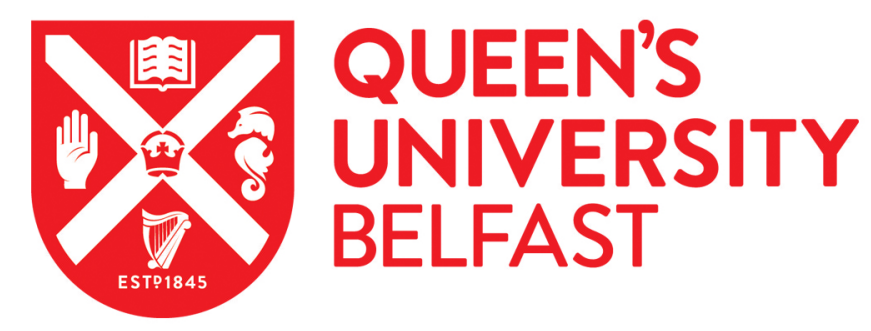

\title{
Diagnostic accuracy of loop-mediated isothermal amplification as a near-patient test for meningococcal disease in children: An observational cohort study
}

Bourke, T., McKenna, J. P., Coyle, P. V., Shields, M. D., \& Fairley, D. J. (2015). Diagnostic accuracy of loopmediated isothermal amplification as a near-patient test for meningococcal disease in children: An observational cohort study. The Lancet Infectious Diseases, 15(5), 552-558. https://doi.org/10.1016/S1473-3099(15)70038-1

Published in:

The Lancet Infectious Diseases

Document Version:

Early version, also known as pre-print

Queen's University Belfast - Research Portal:

Link to publication record in Queen's University Belfast Research Portal

Publisher rights

(c) 2015 the author(s)

\section{General rights}

Copyright for the publications made accessible via the Queen's University Belfast Research Portal is retained by the author(s) and / or other copyright owners and it is a condition of accessing these publications that users recognise and abide by the legal requirements associated with these rights.

\section{Take down policy}

The Research Portal is Queen's institutional repository that provides access to Queen's research output. Every effort has been made to ensure that content in the Research Portal does not infringe any person's rights, or applicable UK laws. If you discover content in the Research Portal that you believe breaches copyright or violates any law, please contact openaccess@qub.ac.uk. 
1 Diagnostic accuracy of 'loop mediated isothermal amplification' (LAMP) as a near-

2 patient test for meningococcal disease in children

3

4 Thomas W. Bourke MD ${ }^{\mathrm{a}, \mathrm{c}}$

5 James P. McKenna PhD ${ }^{b}$

6 Prof Peter V. Coyle MD ${ }^{\mathrm{b}}$

7 Prof Michael D. Shields MD ${ }^{a, c}$

8 Derek J. Fairley $\mathrm{PhD}^{\mathrm{a}, \mathrm{b}}$

9

$10{ }^{a}$ Centre for Infection and Immunity, Queen's University Belfast, Belfast, UK

$11{ }^{\mathrm{b}}$ Regional Virus Laboratory, Department of Microbiology, Belfast Health \& Social Care

12 Trust, Belfast, UK

$13{ }^{\mathrm{c}}$ Royal Belfast Hospital for Sick Children, Belfast Health \& Social Care Trust, Belfast, 14 UK

15

16 Address correspondence to:

17

18 Professor Michael D. Shields,

19 Centre for Infection \& Immunity,

$20 \quad$ Health Sciences Building,

2197 Lisburn Road, Belfast, BT9 7AE, UK

$22 \quad$ Email: m.shields@qub.ac.uk

23

24 


\section{Abstract}

27 Background: Diagnosis of meningococcal disease relies on recognition of clinical signs and symptoms that are notoriously non-specific, variable, and often absent in the early stages of disease. Laboratory testing serves primarily to confirm the clinical diagnosis, as results may not be available for many hours or days. A method called 'loop mediated isothermal amplification' (LAMP) has previously been shown to be fast and effective for molecular detection of meningococcal DNA in clinical specimens.

Methods: We evaluated the diagnostic accuracy (sensitivity and specificity) of

34 meningococcal LAMP as a near-patient test in the emergency department of a large 35 children's hospital. Respiratory (nasopharyngeal swab) and blood specimens from children with suspected meningococcal infection were tested using a near-patient LAMP protocol and compared to reference laboratory testing.

Findings: Combined testing of respiratory and blood specimens using LAMP was accurate (sensitivity 89\%; specificity 100\%; PPV 100\%; NPV 98\%) and diagnostically useful

40 (positive and negative likelihood ratios of $\infty$ and 0.11 respectively). The median time required for near-patient testing was 1 hour 26 minutes from sample to result. laboratory facilities, and near-patient testing using this method is both feasible and effective.

44 In contrast to current UK NICE guidance, we found molecular testing of non-invasive respiratory specimens from children to be diagnostically accurate and clinically useful.

46 Funding: Health \& Social Care Research and Development, Public Health Agency, Northern 47 Ireland. 
51 Clinical diagnosis is the current standard for identifying invasive Neisseria meningitidis

52 infection (meningococcal disease, MD) despite the signs and symptoms being notoriously 53 non-specific, especially in young children. ${ }^{1,2}$ Although MD can progress very quickly, the 54 typical signs and symptoms (if present) do not appear until a median of 13 to 22 hours after 55 the first onset of symptoms, and half of children who present to their GP in the early stages of MD are not referred or admitted to hospital at the first presentation. ${ }^{3}$ Conversely, fear of missing the diagnosis also leads to overtreatment. ${ }^{4}$ Most children admitted and treated as suspected MD turn out to have a less serious infection, and a previous study in our hospital

59 found two thirds of children treated as possible MD had an alternative diagnosis. ${ }^{5}$ Although introduction of an effective vaccine against serogroup B meningococcus should reduce the prevalence of meningococcal infection, ${ }^{6}$ there is considerable scope to improve diagnostic testing.

63

64 Laboratory tests serve only to confirm the clinical diagnosis, as definitive results are rarely 65 available in time to influence clinical decision making. ${ }^{7}$ We previously developed a rapid and effective molecular test based on 'loop-mediated isothermal amplification' (LAMP) to detect meningococcal DNA (ctrA gene) in clinical specimens. ${ }^{8}$ This assay gives equivalent performance to the current UK reference molecular test $\left(\operatorname{TaqMan}^{\circledR}\right.$ real-time $\mathrm{PCR}^{9-12}$ ) but is faster and less expensive. In the UK, the National Institute of Health and Care Excellence

70 (NICE) recognises that molecular (PCR) testing of sterile site specimens for meningococcus 71 is very effective, but also notes that PCR is not available in most hospitals due to resource 72 limitations. $^{13}$ 
74 Detection of meningococcal DNA in a sterile site (blood or CSF) specimen confirms a 75 diagnosis of invasive $\mathrm{MD},{ }^{13}$ but this testing assumes that a level of clinical suspicion is 76 already present. Collecting blood or CSF specimens from every patient with pyrexia but 77 without the classical features of meningococcal infection is neither feasible nor desirable. 78 Nevertheless, this group will include the small number of patients who have early-stage MD 79 and who are at risk of being falsely reassured. Obtaining a nasopharyngeal specimen is 80 relatively non-invasive, although current advice in the UK is not to test these for

81 meningococcus due to the risk of detecting asymptomatic carriage. ${ }^{13} \mathrm{We}$ have previously 82 shown, using sensitive and specific PCR methods, that carriage rates of pathogenic 83 (capsular) meningococci in young children are very low. ${ }^{14}$ Our previous study found 84 molecular testing of throat swabs had a sensitivity of $81 \%$ and a specificity of $100 \%$ for 85 diagnosis of MD. If a suitable molecular assay to detect capsular meningococci is used, we propose that near-patient testing of non-invasive respiratory specimens can provide valuable information to clinicians.

88

89 We report here a study to evaluate the diagnostic accuracy of meningococcal LAMP as a near-patient test on respiratory (combined nasal and throat swab) and blood specimens in patients with suspected MD. Respiratory, blood and CSF specimens were tested in the laboratory using a reference PCR assay. Conventional blood and CSF culture methods were used, as per normal clinical practice, and the diagnostic performance of standard 'non

94 specific' laboratory tests was evaluated for comparison. 
Materials and methods

97 Study design, inclusion criteria and specimens

98

99 The study was approved by the Office for Research Ethics Committee Northern Ireland 100 [reference 09/NIR02/43]. Clinical specimens were collected as per normal Royal Belfast 101 Hospital for Sick Children (RBHSC) practice, with near-patient testing of aliquots as described below. Written informed consent was obtained from the parents of all study participants before their near-patient test results were included in the study.

104

105 The study was designed as a prospective cohort study of diagnostic accuracy. Children (aged 0 to 13 years) presenting to the emergency department between November 2009 and January

1072012 were eligible for inclusion. Patients with suspected meningitis or septicaemia entered 108 a clinical care pathway and had a standard 'meningococcal pack' of investigations (Table 109 1). This group included those whom the admitting doctor suspected might have MD: 110 children with fever, unwell appearance, non-blanching rash, signs of meningitis or signs of septicaemia.

115 Near-patient testing of clinical specimens (nasopharyngeal swabs and EDTA blood) was 116 done in a room adjacent to the pediatric emergency department. Further details of the near117 patient LAMP protocol are given in the appendix. In brief, DNA was extracted from blood 118 and respiratory (combined nasopharyngeal swab) specimens using a simple commercially 119 available DNA extraction system. DNA extracts were analysed immediately using LAMP 
reagents that were prepared in advance and stored frozen in the emergency department.

121 Unlike molecular amplification methods such as PCR, positive LAMP reactions can be

122 identified by visual inspection of the reaction tubes after incubation at an appropriate

123 temperature. In this study, four near-patient LAMP tests were run for each patient (EDTA

124 blood; combined nasopharyngeal swab; positive control; negative control) and test results

125 were read after incubation at $63^{\circ} \mathrm{C}$ for 60 minutes.

126

127 Reference laboratory tests

128

129 Details of the gold standard reference laboratory tests are given in the appendix.

130

131 Statistical methods

133 Laboratory confirmed MD was defined as: “A clinically compatible case plus isolation of

$134 N$. meningitidis or detection of $N$. meningitidis DNA from a normally sterile site (blood or

135 CSF)". ${ }^{15}$ Likelihood ratios (LR), sensitivity, specificity and positive and negative predictive 136 values (PPV, NPV) were calculated for near-patient LAMP testing of nasopharyngeal 137 specimens, blood specimens and both tests combined for diagnosis of MD. 95\% confidence 138 intervals were calculated for estimates of diagnostic accuracy. Staff in both the MRU and 139 BHSCT laboratories were blinded to the results of near-patient testing. ${ }^{16}$

141 Role of the funding source

142

143 The funding sponsor had no role in the study design, data interpretation or decision to submit 144 for publication. 
145

146 


\section{Results}

148

149 Laboratory confirmed meningococcal disease

150

151 In total, 161 patients had a 'meningococcal pack' of investigations and were tested.

152 Subsequently, one declined consent, and a further 12 were not approached for consent (two

153 died; one child protection case; two discharged early; seven transferred to other units). The

154 remaining 148 patients were consented into the study. Most were under five years old

155 (median 11 months; range 17 days - 12.5 years) and 57\% were male gender. 27/148 (18\%)

156 were found to have laboratory confirmed MD, and 121/148 (82\%) had other conditions

157 (Table 2). Only 7/27 (26\%) of the children with confirmed MD had meningococcus isolated

158 in blood culture, and only one of these was positive by blood culture alone. 26/27 (96\%)

159 were positive for meningococcal DNA in their blood by PCR. 8/27 (30\%) of the children

160 with confirmed MD had a lumbar puncture to obtain CSF (Table 3) and all were culture

161 negative. Seven had CSF analysed for meningococcal DNA in the laboratory and 6/7 (86\%)

162 were positive. Antibiotics had been given prior to lumbar puncture to all patients in our 163 study, giving CSF culture a sensitivity of zero. In contrast, molecular testing of CSF was

164 clearly useful, although none of the patients here were positive by molecular testing of CSF 165 alone.

166

16711 children with laboratory confirmed MD had viral co-infections diagnosed by routine 168 molecular virology testing, and three were infected with more than one virus. One child was 169 positive in blood for enterovirus, seven were positive for respiratory viruses (picornavirus, 170 rhinovirus, enterovirus, influenza A, bocavirus) and three were positive in stool specimens 171 (astrovirus, rotavirus or picornavirus). Four of the children who did not have MD had other 
172 invasive bacterial infections confirmed by routine bacteriology testing. Two had $S$. 173 pneumoniae in blood culture; one had E. coli in blood, urine and CSF culture; one had S. 174 agalacticae in blood culture.

175

176 Near-patient testing using meningococcal LAMP

177

178 The median time taken to complete the near-patient meningococcal LAMP tests (from 179 starting extraction to reading results) was 1 hour 26 minutes. Results of near-patient testing 180 using meningococcal LAMP are shown in Table 4. 141/148 children had a combined nasal 181 and throat swab taken and tested using the near-patient LAMP protocol. The performance 182 of near-patient nasopharyngeal testing (Table 4A) was as follows: sensitivity $84 \%$ (95\% CI 183 65-94); specificity 100\% (95\% CI 98-100); PPV 100\% (95\% CI 81-100); NPV 97\% (95\% 184 CI 91-99); positive LR $\infty$; negative LR 0.16 (95\% CI 0.07-0.39). 144/148 children had 185 sufficient blood taken for testing using the near-patient LAMP protocol. The performance 186 of near-patient blood testing (Table 4B) was: sensitivity 84\% (95\% CI 72-99); specificity 187 100\% (95\% CI 93-100); PPV 100\% (95\% CI 75-100); NPV 97\% (95\% CI 90-99); positive 188 LR $\infty$; negative LR 0.16 (95\% CI 0.01-0.42). All 148 children had at least one specimen 189 (nasopharyngeal swab and/or blood) analysed in the near patient setting. If patients who 190 were positive by either of the near-patient tests were considered to be positive, the combined 191 performance of near-patient LAMP testing of blood and/or nasopharyngeal specimens 192 (Table 4C) was: sensitivity 89\% (95\% CI 72-96); specificity 100\% (95\% CI 97-100); PPV 193 100\% (95\% CI 83-100); NPV 98\% (95\% CI 93-99); positive LR $\infty$; negative LR 0.11 (95\% 194 CI 0.04-0.32). 
196 Three patients with laboratory confirmed MD were negative by both near-patient LAMP

197 tests. Two had classical signs and symptoms of MD, and the third was an atypical 198 presentation; a two year-old girl recalled to the hospital when a blood culture taken the 199 previous day grew N. meningitidis. At first presentation, her WCC and CRP were normal, 200 and she was discharged on oral amoxicillin after a period of observation. When recalled 20 201 hours later, her parents reported that she had remained well since discharge. Physical 202 examination revealed no meningism, normal perfusion and a few non-blanching spots on 203 her abdomen. Her WCC had risen to $28.2 \times 10^{9} / 1$ and CRP to $211 \mathrm{mg} / 1$. Her nasopharyngeal 204 and blood specimens were both PCR positive for meningococcal DNA.

206 'Non-specific' laboratory tests

208 The results of 'non-specific' laboratory tests are shown in Table 5. Near-patient LAMP 209 testing was substantially more accurate than any of the routinely used non-specific tests for 210 diagnosis of MD. 


\section{Discussion}

213 Compared to the gold standard laboratory diagnosis of MD, the near-patient LAMP assays 214 gave impressive results. Comparison to WCC and CRP indicates that LAMP tests are more 215 useful than conventional non-specific tests for both ruling in and ruling out meningococcal 216 disease. In addition to being accurate, this study demonstrates that molecular testing in the emergency department is feasible. The LAMP assay performed similarly well in the nearpatient setting and the laboratory setting, using different extraction protocols and different operators, with results typically available in less than two hours. We describe the accuracy

220 of meningococcal LAMP in terms of likelihood ratios, which are not affected by disease

221 prevalence. ${ }^{17}$ Likelihood ratios greater than 10 and less than one are considered strong 222 evidence for the value of a diagnostic test. ${ }^{18}$ By this metric, the near-patient LAMP assay 223 used here is clearly useful for diagnosis of MD.

225 There is likely to be resistance to testing of nasopharyngeal specimens for meningococcus 226 because of the potential to detect asymptomatic carriage. No carriage was detected during 227 this study. Although a larger study may have identified some carriage and reduced the specificity from $100 \%$, the data supports our previous conclusion that molecular testing of nasopharyngeal specimens in very young children is diagnostically useful, and generally not 230 confounded by carriage. ${ }^{14}$ A relatively non-invasive nasopharyngeal meningococcal LAMP 231 test on children presenting with 'fever without source' where the prevalence of meningococcal disease is very low should perform with reasonable accuracy. In this context a positive result is strongly suggestive of disease and could prompt further investigation and

234 treatment. Patients with a negative result could be discharged safely after a period of 235 observation, subject to the universal advice to re-attend if deterioration occurs. Most children 
with fever still present to their general practitioner where the prevalence of meningococcal

237 disease is much lower still. ${ }^{19,20}$ A non-invasive test with a strong negative likelihood ratio used in combination with careful clinical assessment is likely to be even more valuable in this context.

241 Our data support the cost minimisation analysis conducted by NICE which suggests that rapid ( $<24$ hours) access to molecular (PCR) test results can reduce costs through earlier discharge of the relatively well patients. NICE conclude that "the infrastructure does not

244 currently exist to support such a strategy and is unlikely to exist within the next few years". ${ }^{13}$

245 Meningococcal LAMP could be used immediately in any small to medium sized hospital 246 with access to a basic laboratory to give results within a few hours. The meningococcal 247 LAMP assay described here, used in combination with careful clinical assessment, could 248 have led to earlier discharge of a significant number of 'not unwell' patients in this study 249 who did not have MD.

Many of the MD cases in this study also had viral co-infections. As reported previously ${ }^{21}$ we note that in the absence of rapid molecular testing for bacterial pathogens, positive molecular virology test results for viruses such as enterovirus or picornavirus should not reassure clinicians that an unwell patient does not have MD.

256 One limitation of our study was that the proportion of positive cases (19\%) was lower than 257 a previous study in our unit (33\%) although this confirms there was no bias towards more 258 severe case. The blood culture positive rate of $\sim 25 \%$ is similar to previous studies ${ }^{22,23}$ and 259 the 'PCR only' case ascertainment (77\%) is slightly higher than reports from the national 260 reference laboratory ${ }^{23}$ indicating a representative case mix of patients. We experienced 
historically low levels of meningococcal disease during this study, with only 36 confirmed cases in Northern Ireland in 2011 compared with 94 cases in 2004. This has resulted in wider confidence intervals for our estimates of diagnostic accuracy. A small number of discordant

264 test results were seen, which illustrates the problems that are common with definitive 265 diagnosis of MD, even using the best available reference laboratory tests. This study was not designed to investigate whether early availability of definitive test results could influence patient management and improve clinical outcomes. A larger multi-centre trial of nearpatient LAMP for children presenting with 'fever without source' could address this important question. Since this work was completed, the meningococcal LAMP test has been

270 developed further, using freeze-dried reagents, real-time detection and improved 271 amplification chemistry to improve sensitivity and reduce the assay time to below 20 272 minutes.

274 This study is the first to evaluate a rapid near-patient molecular diagnostic test for MD, and 275 also the first demonstration of near-patient testing using LAMP in a UK hospital. The data 276 support our initial hypothesis that testing of nasal and throat swabs using meningococcal 277 LAMP in a near-patient setting is useful as a rapid diagnostic test for $N$. meningitidis. LAMP testing of nasopharyngeal or blood specimens in a near-patient setting was both feasible and accurate, and combining the results for both specimen types further increased the diagnostic accuracy. These data suggest the ability to rapidly detect a pathogenic (capsular) strain of $N$. meningitidis in the nasopharynx of a febrile child whose parents are sufficiently concerned to seek medical advice can give clinically useful information. Current advice from Public

283 Health England (PHE) and NICE on testing of respiratory specimens is inconsistent. PHE 284 recommends culture of nasopharyngeal swabs from suspected cases. ${ }^{24}$ In contrast, NICE 285 explicitly recommend that nasopharyngeal specimens are not tested, by any method. ${ }^{13}$ The 
286 potential to diagnose and treat life-threatening invasive disease early outweighs the small 287 risk of detecting asymptomatic carriage in young children, and this should be considered in reviewed NICE guidance.

289

\section{Research in context panel}

Systematic review

293

294 A PubMed search on Dec 5th, 2014, with the terms ((near patient AND meningococc*) OR 295 (near patient AND meningitidis)) found 16 papers, none related to near-patient testing. 296 Searching with the terms ((point of care AND meningococc*) OR (point of care AND meningitidis)) found 7 papers, two reporting methods suitable for near-patient testing ${ }^{8,25}$.

One of these $\mathrm{e}^{25}$ describes a LAMP-based testing system that "has potential" for point of care testing, but presents no clinical validation data in either laboratory or near-patient settings. The other ${ }^{8}$ is the assay used here, and is the first published and clinically validated LAMP assay for rapid detection of meningococcal DNA. This study is the first to evaluate such an assay for near-patient testing.

304 Interpretation

306 There is currently no effective diagnostic test for meningococcal disease ${ }^{2}$. Cases with clear signs and symptoms are (or should be) diagnosed clinically, and laboratory testing serves mainly to confirm the clinical diagnosis. The decision to obtain and test sterile site specimens (blood or CSF) presumes that a clinical suspicion of serious invasive disease 
already exists. No clinician who suspects meningococcal disease will wait for laboratory results before starting antibiotic treatment, so testing simply confirms an existing diagnosis.

313 Unfortunately, in the absence of definitive diagnostic testing, children with non-specific 314 symptoms who are actually in the early stages of meningococcal disease are frequently 315 overlooked. Half of children with confirmed meningococcal infection have been seen by a 316 healthcare professional and either falsely reassured or discharged in the hours leading up to

317 their clinical diagnosis and treatment. ${ }^{3}$ It seems self-evident that identifying and treating 318 these children earlier could improve clinical outcomes, although there is no data to confirm 319 this. Without an accurate diagnostic test for meningococcal disease, ideally delivered at 320 point-of-care, there is little prospect of identifying these cases earlier in a clinical trial.

322 This study confirms that molecular testing using LAMP to detect meningococcus is highly 323 sensitive and specific, and demonstrates this for the first time in the context of near-patient 324 testing in the emergency department. Molecular tests to detect this pathogen should not be 325 confined to the reference laboratory.

\section{Acknowledgements}

331 We thank the staff of the Regional Virus Laboratory and the Pediatric Emergency 332 Department for their support and assistance. This study was funded by a research fellowship 333 from Health \& Social Care Research and Development, Public Health Agency, Northern 334 Ireland. 


\section{Declaration of interests}

337 TWB received a grant from the Health \& Social Care Research and Development, Public 338 Health Agency, Northern Ireland. JPM, PVC and DJF hold share options in Hibergene 339 Diagnostics Ltd. JPM, PVC and DJF hold patent US 8465927 B2 related to the 340 meningococcal LAMP assays used in this study which is licensed to Hibergene Diagnostics 341 Ltd. MDS has nothing to disclose.

342

343 


\section{References}

345

3461 Bourke TW, Fairley DJ, Shields MD. Rapid diagnosis of meningococcal disease.

347 Expert Rev. Anti Infect. Ther. 2010; 8: 1321-3.

348

3492 Curtis S, Stobart K, Vandermeer B, Simel D, Klassen T. Clinical features

350 suggestive of meningitis in children: a systematic review of prospective data. Pediatrics

$351 \quad 2010 ; 126: 952-60$.

352

3533 Thompson MJ, Ninis N, Perera R, et al. Clinical recognition of meningococcal

354 disease in children and adolescents. Lancet 2006; 367: 397-403.

355

3564 O’Donnell DR. Recognising severe infection. Arch. Dis. Child. 2010; 95: 957-8.

3585 Dunlop K, Coyle PV, Jackson P, Patterson CC, Shields MD. Respiratory viruses do 359 not trigger meningococcal disease in children. J. Infect. 2007; 54: 454-8.

360

3616 Vesikari T, Esposito S, Prymula R, et al. Immunogenicity and safety of an

362 investigational multicomponent, recombinant, meningococcal serogroup B vaccine

363 (4CMenB) administered concomitantly with routine infant and child vaccinations: results

364 of two randomised trials. Lancet 2013; 381: 825-35.

365

3667 Bissonnette L, Bergeron MG. Infectious disease management through point-of-care 367 personalized medicine. J. Pers. Med. 2012; 2: 50-70. 
3698 McKenna JP, Fairley DJ, Shields MD, Cosby S, Wyatt D, McCaughey C, Coyle

370 PV. Development and clinical validation of a loop-mediated isothermal amplification

371 method for the rapid detection of Neisseria meningitidis. Diagn. Microbiol. Infect. Dis.

$372 \quad 2011 ; 69: 137-44$.

373

3749 Guiver M, Borrow R, Marsh J, et al. Evaluation of the Applied Biosystems 375 automated Taqman polymerase chain reaction system for the detection of meningococcal 376 DNA. FEMS Immunol. Med. Microbiol. 2000; 28: 173-9.

377

37810 Carrol ED, Thomson APJ, Shears P, Gray SJ, Kaczmarski EB, Hart C.

379 Performance characteristics of the polymerase chain reaction assay to confirm clinical 380 meningococcal disease. Arch. Dis. Child. 2000; 83: 271-3.

381

38211 Corless CE, Guiver M, Borrow R, Edwards-Jones V, Fox A Kaczmarski EB.

383 Simultaneous detection of Neisseria meningitidis, Haemophilus influenzae, and

384 Streptococcus pneumoniae in suspected cases of meningitis and septicemia using real-time 385 PCR. J. Clin. Micro. 2001; 39: 1553-8.

386

387 Bryant PA, Li HY, Zaia A, Griffith J, Hogg G, Curtis N, Carapetis J. Prospective 388 study of a real-time PCR that is highly sensitive, specific, and clinically useful for 389 diagnosis of meningococcal disease in children. J. Clin. Micro. 2004; 42: 2919-25.

39113 National Institute for Health and Care Excellence. 2010. NICE Guidance CG102:

392 Bacterial meningitis and meningococcal septicaemia: Management of bacterial meningitis 
and meningococcal septicaemia in children and young people younger than 16 years in

394 primary and secondary care. https://www.nice.org.uk/guidance/cg102

395

39614 Dunlop K, Coyle, P, Mitchell S, Fairley D, O’Neill H, Jackson P, Shields MD.

397 Molecular testing of respiratory swabs aids early recognition of meningococcal disease in 398 children. Diagn. Microbiol. Infect. Dis. 2011; 70: 427-34.

399

40015 Trotter CL, Chandra M, Cano R, et al. A surveillance network for meningococcal 401 disease in Europe. FEMS Microbiol. Rev. 2007; 31: 27-36.

402

40316 Bossuyt PM, Reitsma JB, Bruns DE, et al. Towards complete and accurate 404 reporting of studies of diagnostic accuracy: the STARD initiative. Br. Med. J. 2003; 326: $40541-4$.

406

40717 Moayyeri A. Likelihood ratios for dynamic decision making. Br. Med. J. 2004; 408 329: 168.

409

41018 Deeks JJ, Altman DG. Diagnostic tests 4: likelihood ratios. Br. Med. J. 2004; 329: $411 \quad 168-9$.

41319 Maguire S, Ranmal R, Komulainen S, et al. Which urgent care services do febrile 414 children use and why? Arch Dis Child. 2011; 96: 810-6.

415

41620 Van den Bruel A, Haj-Hassan T, Thompson M, Buntinx F, Mant D; European 417 Research Network on Recognising Serious Infection investigators. Diagnostic value of 
418 clinical features at presentation to identify serious infection in children in developed

419 countries: a systematic review. Lancet 2010; 375: 834-45.

420

42121 Basmaci R, Mariani P, Delacroix G, et al. Enteroviral Meningitis Does Not

422 Exclude Concurrent Bacterial Meningitis. J Clin Microbiol. 2011; 49: 3442-3.

423

42422 Ragunathan L, Ramsay M, Borrow R, Guiver M, Gray S Kaczmarski EB. Clinical

425 features, laboratory findings and management of meningococcal meningitis in England and

426 Wales: Report of a 1997 survey. J. Infect. 2000; 40: 74-9.

427

42823 Meningococcal Reference Unit, Gray SJ, Trotter CL, et al. Epidemiology of

429 meningococcal disease in England and Wales 1993/94 to 2003/04: contribution and

430 experiences of the Meningococcal Reference Unit. J. Med. Microbiol. 2006; 55: 887-96.

431

432 Health Protection Agency Meningococcus and Haemophilus Forum. 2012.

433 Guidance for public health management of meningococcal disease in the UK.

434 https://www.gov.uk/government/publications/meningococcal-disease-guidance-on-public-

435 health-management

436

43725 Dou M, Dominguez DC, Li X, Sanchez J, Scott G. A versatile PDMS / paper hybrid 438 microfluidic platform for sensitive infectious disease diagnosis. Anal Chem. 2014; 86: 797843986 
TABLE 1 'Meningococcal pack' investigations

\begin{tabular}{|c|c|c|}
\hline Specimen & Volume & Test \\
\hline Whole blood & At least $1 \mathrm{ml}$ & Blood culture, collected prior to antibiotic therapy if possible \\
\hline EDTA blood & $1 \mathrm{ml}$ & Meningococcal ctrA TaqMan ${ }^{\circledR}$ qPCR \\
\hline EDTA blood & $1 \mathrm{ml}^{\mathrm{a}}$ & Enterovirus / picornavirus RT-qPCR \\
\hline EDTA blood & $0.5 \mathrm{ml}$ & Full blood count \\
\hline Clotted blood & $0.5 \mathrm{ml}$ & $\begin{array}{l}\text { Routine biochemistry: renal function; electrolytes; calcium; } \\
\text { magnesium; C-reactive protein (CRP) }\end{array}$ \\
\hline Heparinised blood & $1.4 \mathrm{ml}$ & Coagulation screen \\
\hline EDTA blood & $2 \mathrm{ml}$ & Routine haematology / blood group \\
\hline $\begin{array}{l}\text { Combined nasal \& } \\
\text { throat swab }\end{array}$ & $1 \mathrm{ml}^{\mathrm{a}}$ & $\begin{array}{l}\text { Swabs combined in eNAT transport medium; meningococcal } \\
\operatorname{ctrA~TaqMan}{ }^{\circledR} \mathrm{qPCR} / \text { LAMP and viral screen }\end{array}$ \\
\hline CSF (optional) & $1 \mathrm{ml} l^{\mathrm{b}}$ & Routine biochemistry, WCC, culture and viral screen \\
\hline
\end{tabular}

${ }^{\mathrm{a}} 0.2 \mathrm{ml}$ aliquots of these specimens were processed using the near-patient LAMP protocol.

${ }^{b}$ Lumbar puncture to obtain CSF is done only if clinically indicated. 
TABLE 2 Reference laboratory results for confirmed MD cases ( $\mathrm{n}=27$ )

\begin{tabular}{|c|c|c|c|c|c|}
\hline \multirow[b]{2}{*}{ Patient no. } & \multicolumn{2}{|c|}{ Reference tests to confirm MD } & \multirow[b]{2}{*}{ Serogroup $^{a}$} & \multicolumn{2}{|c|}{ Laboratory LAMP tests } \\
\hline & Blood culture & Blood $\mathrm{PCR}^{\mathrm{a}}$ & & Swab LAMP & Blood LAMP \\
\hline 3 & + & + & $\mathrm{B}$ & + & + \\
\hline 4 & - & + & $\mathrm{B}$ & + & + \\
\hline 10 & - & + & $\mathrm{B}$ & + & + \\
\hline 13 & - & + & $\mathrm{B}$ & + & + \\
\hline 21 & - & + & $\mathrm{B}$ & + & NA \\
\hline 29 & + & + & $\mathrm{B}$ & - & + \\
\hline 30 & - & + & $\mathrm{B}$ & + & + \\
\hline 36 & - & + & $\mathrm{B}$ & + & + \\
\hline 73 & - & + & $\mathrm{B}$ & + & + \\
\hline 85 & + & + & $\mathrm{Y}$ & + & + \\
\hline 87 & - & + & B & + & + \\
\hline 91 & + & + & B & + & + \\
\hline 93 & - & + & B & + & + \\
\hline 94 & - & + & B & + & + \\
\hline 106 & - & + & B & + & + \\
\hline 110 & - & + & B & + & + \\
\hline 114 & + & + & B & + & + \\
\hline 116 & - & + & B & + & + \\
\hline 132 & - & + & B & + & + \\
\hline 133 & - & + & B & + & + \\
\hline 134 & - & + & B & + & + \\
\hline 135 & - & + & B & NA & + \\
\hline 145 & - & + & B & + & + \\
\hline 146 & + & + & B & + & + \\
\hline 157 & - & + & B & + & + \\
\hline 158 & - & + & B & + & + \\
\hline 160 & + & + & B & NA & + \\
\hline
\end{tabular}

${ }^{a}$ PCR testing (ctrA TaqMan ${ }^{\circledR}$ assay and specific meningococcal serogrouping qPCR assays) were performed in Manchester (MRU), Belfast (BHSCT) or both.

${ }^{\mathrm{b}}$ Combined nasopharyngeal swab; NA = specimen not available 
TABLE 3 Results for patients with confirmed meningococcal disease where CSF was examined

\begin{tabular}{ccccc}
\hline Patient no. & $\begin{array}{c}\text { Time from } \\
\text { treatment (hrs) }\end{array}$ & CSF culture & CSF LAMP & CSF TaqMan \\
\hline 10 & 22 & - & + & + \\
13 & 126 & - & - & - \\
36 & 46 & - & + & + \\
73 & 16 & - & + & $\mathrm{ND}$ \\
94 & 42 & - & + & + \\
114 & 2 & - & + & + \\
145 & 48 & - & + & + \\
158 & 44 & - & $\mathrm{ND}$ & $\mathrm{ND}$ \\
\hline
\end{tabular}

ND, not done 
TABLE 4 Results of near-patient meningococcal LAMP testing A: Nasopharyngeal specimens $(n=141)$

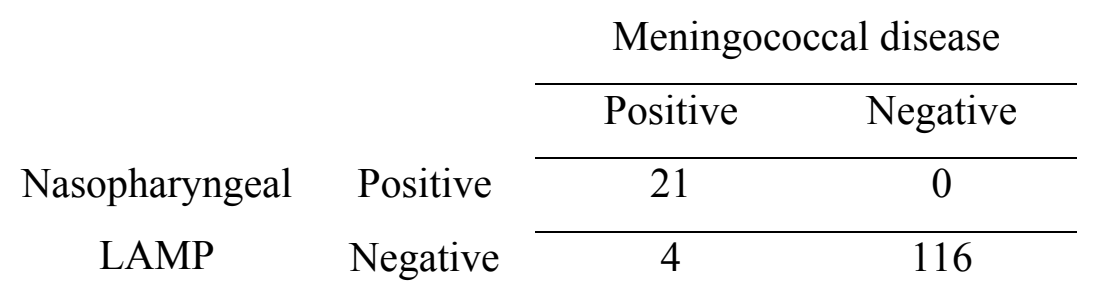

B: EDTA blood specimens $(\mathrm{n}=144)$

\begin{tabular}{cccc} 
& & \multicolumn{2}{c}{ Meningococcal disease } \\
\cline { 3 - 4 } Blood & Positive & Positive & Negative \\
\cline { 3 - 4 } LAMP & Negative & 4 & 0 \\
\cline { 3 - 4 } & & &
\end{tabular}

C: Combined results for both specimen types $(n=148)$

\begin{tabular}{cccc} 
& & \multicolumn{2}{c}{ Meningococcal disease } \\
\cline { 3 - 4 } Nasopharyngeal & & Positive & Negative \\
\cline { 3 - 4 } \&/or blood & Positive & 24 & 0 \\
\cline { 3 - 3 } LAMP & Negative & 3 & 121
\end{tabular}


TABLE 5 Results of 'non-specific' laboratory tests

\begin{tabular}{lcccc}
\hline Test $^{\mathrm{a}}$ & $\begin{array}{c}\text { Sensitivity } \\
(95 \% \mathrm{CI})\end{array}$ & $\begin{array}{c}\text { Specificity } \\
(95 \% \mathrm{CI})\end{array}$ & $\begin{array}{c}\text { Positive LR } \\
(95 \% \mathrm{CI})\end{array}$ & $\begin{array}{c}\text { Negative LR } \\
(95 \% \mathrm{CI})\end{array}$ \\
\hline $\mathrm{CRP}>10 \mathrm{mg} / \mathrm{l}$ & $93 \%$ & $64 \%$ & 2.60 & 0.11 \\
& $(77$ to 98$)$ & $(55$ to 72$)$ & $(1.99-3.39)$ & $(0.03-0.44)$ \\
$\mathrm{CRP}>60 \mathrm{mg} / 1$ & $63 \%$ & $91 \%$ & 7.43 & 0.41 \\
& $(44$ to 78$)$ & $(85$ to 95$)$ & $(3.84-14.37)$ & $(0.25-0.66)$ \\
Abnormal & $78 \%$ & $65 \%$ & 2.22 & 0.34 \\
WCC & $(59$ to 89$)$ & $(56$ to 73$)$ & $(1.61-3.05)$ & $(0.17-0.70)$ \\
Abnormal & $85 \%$ & $52 \%$ & 1.78 & 0.28 \\
neutrophils & $(67$ to 94$)$ & $(43$ to 61$)$ & $(1.39-2.28)$ & $(0.11-0.71)$ \\
\hline
\end{tabular}

${ }^{\mathrm{a}} \mathrm{CRP}$ cutoffs used in the literature range from 8 to 100 (reviewed by NICE ${ }^{13}$ ). Cutoffs used here were selected to optimise sensitivity $(10 \mathrm{mg} / \mathrm{l})$ or specificity $(60 \mathrm{mg} / \mathrm{l})$ based on ROC analysis (data not shown). The reference normal range for WCC in our laboratory is 5 to $13 \times 10^{9} /$. 


\section{Supplementary material}

Near-patient meningococcal LAMP protocol

DNA was extracted from EDTA blood $(200 \mu l)$ and eNat medium $(200 \mu l$, containing combined nasal and throat swabs) using a QuickGene Mini80 system and DB-S DNA kit (Fuji Corporation, Tokyo, Japan). DNA extracts $(100 \mu \mathrm{l})$ were denatured $\left(95^{\circ} \mathrm{C}, 5\right.$ minutes $)$ cooled on ice and tested immediately. Each LAMP reaction $(25 \mu 1)$ comprised $20 \mu 1$ of LAMP mastermix and $5 \mu$ of DNA extract in a $100 \mu$ PCR tube (Abgene Ltd., Epsom, UK). A base LAMP mastermix containing all reagents except DNA polymerase (Bst2.0, 8U/ $\mu \mathrm{l}$; New England Biolabs, Ipswich, USA) and Fluorescence Detection Reagent (FDR; Eiken Chemical Company, Japan) was stored at $-20^{\circ} \mathrm{C}$ in $90 \mu$ aliquots prior to use. Components of the base mastermix (buffer, LAMP primers, betaine, $\mathrm{MgSO}_{4}$ and $\mathrm{dNTPs}$ ) were as described previously. ${ }^{1}$ For each patient, a $90 \mu 1$ aliquot of mastermix was thawed, mixed with DNA polymerase $(5 \mu \mathrm{l})$ and FDR $(5 \mu \mathrm{l})$ and used immediately. Reactions were incubated at $63^{\circ} \mathrm{C}$ using a standard thermal cycler (GeneAmp 9700, Life Technologies, Paisley, UK). After 60 minutes, tubes were visually inspected for a colour change from pale orange to bright yellow/green to identify positive reactions, as illustrated in Figure S1. 


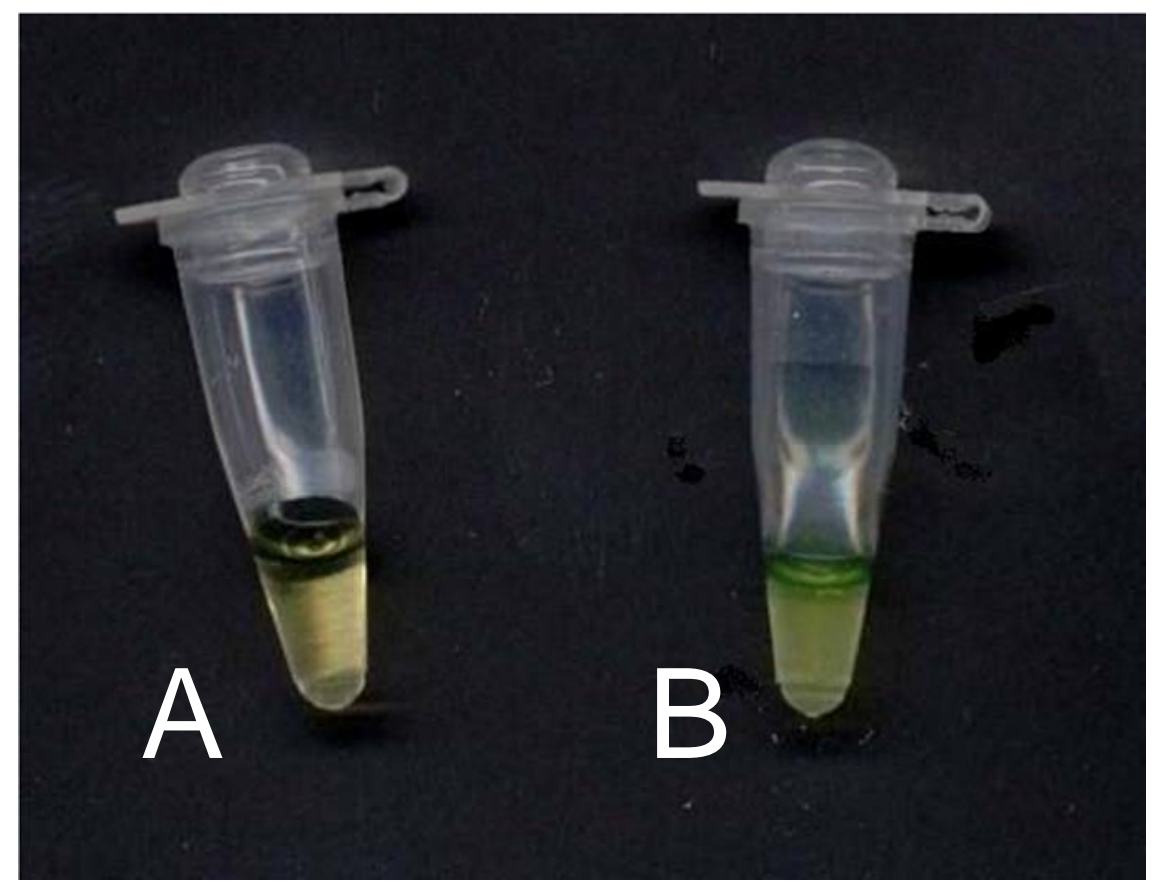

Figure S1. Negative (A) and positive (B) meningococcal LAMP reaction tubes.

Reference laboratory testing

Blood and CSF specimens were cultured according to UK standard methods ${ }^{2,3}$ using an automated BacT/ALERT ${ }^{\circledR}$ 3D Microbial Detection System with BacT/ALERT ${ }^{\circledR}$ FA culture medium (bioMérieux, Marcy l'Etoile, France). Isolates were identified using the VITEK ${ }^{\circledR} 2$ system (bioMérieux) and latex bead agglutination tests (Pastorex Meningitis Kit, Bio-Rad Laboratories Ltd., Hemel Hempstead, UK). PCR testing of EDTA blood was performed by

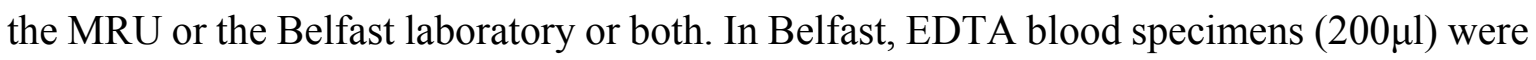
extracted using the QIAsymphony SP automated extraction system and DSP Virus/Pathogen Mini Kit / Complex 200 protocol (Qiagen Ltd., Crawley, UK). PCR assays (10 $\mu 1)$ to detect the meningococcal ctrA gene used Invitrogen Platinum ${ }^{\circledR}$ Quantitative PCR SuperMix-UDG (Life Technologies Ltd., Paisley, UK) with primers and a TaqMan ${ }^{\circledR}$ probe as described 
previously. ${ }^{4}$ An additional reverse primer (5'-TTGCCGCGGATTGGCCACCA-3') was used $^{5}$ to ensure that strains with known mutations in the ctrA gene could be reliably detected. ${ }^{6,7}$ PCR assays were run on a LightCycler ${ }^{\circledR}$ 480II real-time PCR system (Roche Diagnostics Ltd., Burgess Hill, UK) using the following thermal cycling protocol: $50^{\circ} \mathrm{C}(10$ minutes); $95^{\circ} \mathrm{C}$ ( 2 minutes); 45 cycles of $95^{\circ} \mathrm{C}$ (15 seconds) and $60^{\circ} \mathrm{C}$ (30 seconds). $\mathrm{CSF}$ specimens $(200 \mu \mathrm{l})$ were tested in the Belfast laboratory using either PCR or LAMP, as per the near-patient testing protocol, except that DNA extracts were prepared using a QIAamp DNA Blood Mini Kit (Qiagen Ltd.).

Serogrouping PCR

A commercially available real-time PCR kit (Diagenode Diagnostics, Léige, Belgium) was used to determine the genetic capsule type for confirmed cases, with specific primers and TaqMan ${ }^{\circledR}$ probes for capsule biosynthesis genes of the five serogroups (A, B, C, W135, Y) most frequently associated with MD. Reactions $(10 \mu \mathrm{l})$ contained $1 \mathrm{X}$ Platinum ${ }^{\circledR}$ Quantitative PCR SuperMix-UDG (Invitrogen Ltd, Paisley, UK), $4 \mathrm{mM} \mathrm{MgCl} 2,1 \mu \mathrm{l}$ of primer and probe mix, $0.2 \mathrm{mg} / \mathrm{ml}$ bovine serum albumin (Sigma-Aldrich Ltd., Dorset, UK), nuclease free water and $2 \mu \mathrm{l}$ of template DNA. Assays were run on a LightCycler ${ }^{\circledR} 480 \mathrm{II}$ real-time PCR system using the manufacturer's recommended cycling protocol: $50^{\circ} \mathrm{C}(2$ minutes); $95^{\circ} \mathrm{C}$ for $(10$ minutes $) ; 45$ cycles of $95^{\circ} \mathrm{C}$ (10 seconds), $60^{\circ} \mathrm{C}$ (40 seconds) and $72^{\circ} \mathrm{C}$ (1 second). 
References

1 McKenna JP, Fairley DJ, Shields MD, Cosby S, Wyatt D, McCaughey C, Coyle

PV. Development and clinical validation of a loop-mediated isothermal amplification method for the rapid detection of Neisseria meningitidis. Diagn. Microbiol. Infect. Dis. 2011; 69: 137-44.

2 Standards for microbiology investigations, Public Health England. 2014. SMI B 27: Investigation of cerebrospinal fluid. https://www.gov.uk/government/publications/smi$\underline{\text { b-27-investigation-of-cerebrospinal-fluid }}$

3 Standards for microbiology investigations, Public Health England. 2014. SMI B 37: Investigation of blood cultures (for organisms other than Mycobacterium spp.). https://www.gov.uk/government/publications/smi-b-37-investigation-of-blood-culturesfor-organisms-other-than-mycobacterium-species

4 Corless CE, Guiver M, Borrow R, Edwards-Jones V, Fox A Kaczmarski EB. Simultaneous detection of Neisseria meningitidis, Haemophilus influenzae, and Streptococcus pneumoniae in suspected cases of meningitis and septicemia using real-time PCR. J Clin Micro. 2001; 39: 1553-8.

5 Guiver M, Corless CE, Marsh WJ, Gray SJ, Newbold LS, Borrow R and Kaczmarski EB. 2011. Modifications to a Published ctrA PCR Assay for the Improved Non-Culture Confirmation of Meningococcal Disease in England and Wales. Poster 
presented at Meningitis and Septicaemia in Children and Adults, Royal Society of Medicine, London, UK, 8 to 9 November 2011. http://meningitis.org/assets/x/53939

6 Jaton K, Ninet B, Bille J, Greub G. False-Negative PCR Result Due to Gene Polymorphism: the Example of Neisseria meningitidis. J Clin Microbiol. 2010; 48: 45901.

7 Cavrini F, Liguori G, Andreoli A, Sambri V. Multiple Nucleotide Substitutions in the Neisseria meningitidis Serogroup C ctrA Gene Cause False-Negative Detection by Real-Time PCR. J Clin Microbiol. 2010; 48: 3016-8. 